\title{
Étude de la composition corporelle des Meilleurs Lanceurs
}

\section{Algériens.}

\section{Ould-Ahmed.}

Université de Boumerdès, Algrie.

\section{Résumé :}

Le but de cette recherche est d'étudier la composition corporelle des meilleurs lanceurs algériens dans chacune des quatre spécialités de lancers athlétiques.Vingt quatre athlètes composés de cinq lanceurs de poids, sept de disque, 


\section{Introduction :}

L'intérêt porté par plusieurs auteurs à la morphologie du sport de haut niveau, fait apparaitre clairement l'importance de ce facteur comme composante de la performance (Tanner, 1964; Hirata, 1966; Schurch, 1984). D'après Platonov (1984), le niveau atteint par les performances ne rend plus celles-ci accessibles, qu'à des individus doués de qualités morphologiques rares, associées à un très haut niveau de développement des capacités fonctionnelles et mentales.

La composition corporelle correspond à l'analyse du corps humain en compartiments (Barbe et Ritz, 2005). L'influence de la pratique d'une activité sportive sur la composition corporelle d'un individu a fait l'objet de plusieurs recherches et a été démontrée dans plusieurs études (Spenst et coll., 1993; Nindl et coll., 1996; Mavroeidi et Stewart, 2003).

En athlétisme, des études s'intéressant spécifiquement à la morphologie des lanceurs (Morrow et coll., 1982 ; Kidd et Winter, 1983 ; Coh et coll., 2002 ; Kruger et coll., 2006), montrent clairement la grande importance d'une morphologie spécifique dans chaque spécialité de lancers, pour l'atteinte d'un haut niveau de performance.

Concernant les lancers athlétiques en Algérie, aucune étude au préalable n'a été réalisée abordant la morphologie des lanceurs et les particularités morphologiques propres à chaque type de lancers. Notre étude s'intéresse donc à la morphologie des athlètes algériens dans les quatre disciplines de lancer, en particulier à la composition corporelle 
des meilleurs lanceurs dans chacune de ces spécialités, et ce, dans le but de déterminer les caractéristiques de masses musculaire, osseuse et grasse de chaque spécialité de lancer athlétique et les différences de composition corporelle entre chaque type de lancer.

\section{Matériel et méthodes :}

\section{Sujets :}

L'étude a été réalisée sur un échantillon de 24 athlètes mâles représentant les meilleurs lanceurs algériens seniors ayant réalisé les meilleures performances nationales de la saison sportive 2007-2008, repartis en quatre groupes, selon la spécialité de lancer athlétique de chacun : le lancer de poids, de disque, de javelot ou de marteau. Les caractéristiques de notre échantillon sont représentées dans le Tableau 1.

Tableau 1 : Valeurs moyennes des caractéristiques de l'échantillon

\section{Spécialités de lancers}

$\begin{array}{llll}\text { Poids Disque } & \text { Javelot }\end{array}$

n

Age (années) $\quad 23,60 \pm 3,51$

poids ( $\mathrm{kg})$

Stature (cm)

$$
108 \pm 28,78
$$

7

$27,86 \pm 7,52$

$105,57 \pm 20,65$

$110,54 \pm 15,47$
$85,43 \pm 13,11$

\section{5}

$28,80 \pm 8,76$

$21,86 \pm 2,79$

$181,44 \pm 5,92$ 


\section{Matériel :}

Durant la réalisation de nos mesures, les principaux instruments utilisés sont les suivants :

- Une valise anthropométrique de type Siber hegner, contenant : Un anthropomètre du type MARTIN, un compas d'épaisseur à bouts olivaires et un mètre ruban en lin ;

- Une pince à plis (ou caliper) du type Lange (Cambridge Scientific Industries, Cambridge, Maryland);

- Une balance médicale du type SECA.

\section{Méthode d'investigation :}

\section{Méthode anthropométrique :}

Les techniques anthropométriques de base ont été utilisées pour effectuer les mesures et permis de déterminer les paramètres suivants :

- Le poids corporel $(\mathrm{kg})$, déterminé par la balance médicale ;

- La stature $(\mathrm{cm})$, mesurée en utilisant l'anthropomètre ;

- Les diamètres distaux $(\mathrm{cm})$ du bras, de l'avant bras, de la cuisse et de la jambe, mesurés à l'aide du compas d'épaisseur à bouts olivaires ;

- Les circonférences (cm) du bras, de l'avant bras, de la cuisse et de la jambe, déterminés en utilisant le mètre ruban ; 
- Les plis cutanés (mm) bicipital, tricipital, sous scapulaire, du ventre, de la cuisse et de la jambe, mesurés en utilisant la pince a plis ;

Pour l'évaluation des différentes composantes de la masse corporelle (masse musculaire, masse grasse et masse osseuse), nous avons utilisé dans notre étude les formules proposées par Mateigka J. (1921) et qui sont les suivantes :

- Masse musculaire, exprimée en kilogramme, d'après la formule suivante :

$$
M M=6,5 \times T \times R^{2}
$$

Où $\quad \mathrm{MM}$ : masse musculaire en $\mathrm{Kg}$

$\mathrm{T} \quad$ : taille en centimètres

R : la valeur de l'expression :

$$
\mathrm{R}=\left[\left(\sum \text { circonférences }: \text { bras, avant bras, cuisse et jambe }\right) /\right.
$$

$8 \times 3,14]$

- $\left[\left(\sum\right.\right.$ plis cutanés : bras, avant bras, cuisse et jambe $) /$

80]

- Masse osseuse, exprimée en kilogramme, d'après la formule suivante :

$$
M O=1,2 \times T \times O^{2}
$$

Où MO : masse osseuse en $\mathrm{kg}$

$\mathrm{T}$ : taille en $\mathrm{cm}$

$\mathrm{O}$ : la valeur en $\mathrm{cm}$ de l'expression : 


$$
\mathrm{O}=\left(\sum\right. \text { diamètres : distaux bras, avant bras, cuisse et }
$$

jambe)/4.

- Masse grasse (ou adipeuse), exprimée en $\mathrm{kg}$, selon la formule suivante :

$$
M A=1,3 \times S a \times D
$$

Où MA : masse adipeuse en $\mathrm{kg}$

D : la valeur de l'expression :

$\mathrm{D}=1 / 2(\mathrm{~d} 1+\mathrm{d} 2+\mathrm{d} 3+\mathrm{d} 4+\mathrm{d} 5+\mathrm{d} 6) / 12 ;$ où $\mathrm{d} 1$ : plis sous scapulaire,

d2 : pli (biceps +triceps)/2, d3 : pli de l'avant bras, d4 : pli du

ventre,

d5 : pli de la cuisse, d6 : pli de la jambe.

Sa : surface du corps absolue, exprimée en $\mathrm{m}^{2}$, d'après la formule d'Izakson (1958) suivante :

$$
S a=1+([P+(T-160)] / 100)
$$

Où $\mathrm{Sa}$ : surface corporelle absolue exprimée en $\mathrm{m}^{2}$

$$
\mathrm{P} \text { : poids du corps en } \mathrm{Kg}
$$

$\mathrm{T}$ : taille ou stature en $\mathrm{cm}$.

\section{Tests Statistiques}

Nous avons utilisé dans le cadre de notre travail les statistiques descriptives et les tests de comparaison. 
Revue des sciences de l'homme et de la société...................Ould-Ahmed

\section{Résultats :}

Tableau 2.- Composition corporelle des quatre groupes de lancer

\begin{tabular}{|c|c|c|c|c|}
\cline { 2 - 5 } \multicolumn{1}{c|}{} & \multicolumn{3}{c|}{ Spécialités de lancers } \\
\cline { 2 - 5 } \multicolumn{1}{c|}{} & Poids & Disque & Javelot & Marteau \\
\hline Masse musculaire (\%) & $46,92 \pm 5,92$ & $47,74 \pm 3,74$ & $49,68 \pm 4,41$ & $39,72 \pm 3,86$ \\
\hline Masse osseuse (\%) & $13,68 \pm 2,05$ & $15,36 \pm 4,46$ & $14,73 \pm 1,85$ & $11,89 \pm 1,04$ \\
\hline Masse grasse (\%) & $11,62 \pm 6,03$ & $10,56 \pm 3,86$ & $8,75 \pm 5,12$ & $19,17 \pm 3,23$ \\
\hline
\end{tabular}

Les résultats de la composition corporelle des quatre groupes de lancers indiquent que :

- Le groupe des lanceurs de javelot présente le pourcentage de masse musculaire le plus élevé et le pourcentage de masse grasse le moins élevé de l'échantillon.

- Le groupe des lanceurs de marteau présente les pourcentages de masse musculaire et masse osseuse les moins élevé de l'échantillon, tandis que son pourcentage de masse grasse est plus élevé que les autres groupes de lanceurs.

- Le groupe des lanceurs de disque présente le pourcentage de masse osseuse le plus élevé de l'échantillon. 
Tableau 3 : Analyse comparative entre les quatre groupes de lancers

\begin{tabular}{|c|c|l|c|l|c|l|}
\hline \multirow{2}{*}{$\begin{array}{c}\text { Lancers } \\
\text { athlétiques }\end{array}$} & \multicolumn{2}{|c|}{$\begin{array}{c}\text { Masse } \\
\text { musculaire }\end{array}$} & \multicolumn{2}{c|}{$\begin{array}{c}\text { Masse } \\
\text { osseuse }\end{array}$} & \multicolumn{2}{c|}{ Masse grasse } \\
\cline { 2 - 7 } & \multicolumn{2}{|c|}{ t de Student } & \multicolumn{2}{c|}{ t de Student } & \multicolumn{2}{c|}{ t de Student } \\
\hline poids / disque & $-0,272$ & NS & $-0,872$ & NS & 0,346 & NS \\
\hline Poids / javelot & $-0,880$ & NS & $-0,903$ & NS & 0,866 & NS \\
\hline Poids / marteau & 2,279 & NS & 1,741 & NS & $-2,464$ & $*$ \\
\hline disque / javelot & $-0,887$ & NS & 0,345 & NS & 0,748 & NS \\
\hline disque / marteau & 3,595 & $* *$ & 1,982 & NS & $-4,188$ & $* *$ \\
\hline javelot / marteau & 4,151 & $* *$ & 3,373 & $* *$ & $-4,312$ & $* *$ \\
\hline
\end{tabular}

NS : Différence non significative, * différence significative à $\mathrm{p}<0.05$, ** différence significative à $\mathrm{p}<0.01$.

L'analyse comparative entre les quatre groupes de l'échantillon en ce qui concerne leur composition corporelle n'a révélé aucune différence significative entre les groupes de lanceurs de poids, de disque et de javelot.

Par contre, le groupe des lanceurs de marteau présente des différences significatives par rapport aux autres groupes de lanceurs. Entre effet, nos résultats ont montré qu'il existe des différences significatives entre les lanceurs de marteau et de javelot concernant les masses musculaire, osseuse et grasse. Nous observons également que 
les lanceurs de marteau présentent le pourcentage de masse grasse le plus élevé de l'échantillon avec des différences significatives par rapport à chacun des autres groupes de lanceurs.

\section{Discussion :}

Nos résultats ont montré que les lanceurs de marteau présentent la valeur moyenne de pourcentage de masse grasse la plus élevée de notre échantillon, suivis des lanceurs de poids de disque et ensuite des lanceurs de javelot. Ces résultats sont en concordance avec ceux obtenus par Morrow et coll. (1982) concernant les meilleurs lanceurs américains, dans la mesure où ce sont également les lanceur de marteau qui présentent le pourcentage de masse grasse le plus élevé, suivis du poids, du disque, puis du javelot.

Nous avons également constaté à travers l'analyse statistique, des différences significatives entre le groupe des lanceurs de marteau et les autres groupes de lanceurs de notre échantillon, à $\mathrm{p}<0,05$ pour les lanceurs de poids, à $\mathrm{p}<0,01$ pour les lanceurs disque et également pour les lanceurs de javelot.

Concernant la masse osseuse, les lanceurs de disque présentent la valeur moyenne de pourcentage, la plus élevée de notre échantillon, suivis des lanceurs de javelot, de poids et ensuite de marteau, avec notamment, une différence statistiquement significative à $\mathrm{p}<0,01$ entre les lanceurs de javelot et les lanceurs de marteau.

D'après les études de Hirata (1966) et De Garay et coll. (1974), les lanceurs masculins (poids, disque et marteau) aux cours 
des jeux olympiques de Tokyo et de Mexico avaient, en moyenne, 30 $\%$ de graisse. Ces résultats ne concordent pas avec nos valeurs moyennes de masse grasse pour les lanceurs de poids, disque et marteau qui sont nettement inférieures à $30 \%$.

Les résultats de l'étude de wilmore (1983), concernant le pourcentage de masse grasse de lanceurs de poids et de disque de haut niveau, a révélé que les lanceurs de poids présentaient un pourcentage de masse grasse (17 à $20 \%$ ) légèrement supérieur à celui des lanceurs de disque $(16 \%)$. Ces résultats sont en concordance avec ceux obtenus dans cette présente étude, où en effet, avec un pourcentage de masse grasse de 11,62\%, les lanceurs de poids présentent une valeur moyenne légèrement supérieure à celle des lanceurs de disque $(10,56$ $\%)$.

L'étude de Coh et coll. (2008), ayant comme échantillon des lanceurs de poids de niveau mondial, a révélé un pourcentage de masse grasse $(16,95 \%)$ supérieur au pourcentage de masse osseuse $(14,45 \%)$. Ces résultats ne concordent pas avec les résultats de notre étude, nos lanceurs de poids présentant un pourcentage de masse grasse $(11,62 \%)$ inférieur au pourcentage de masse osseuse $(13,68$ $\%)$.

L'étude de Kruger et coll. (2006) présentent les pourcentages de masse musculaire $(55,8 \%)$, de masse grasse $(11,9 \%)$ et masse osseuse $(11,7 \%)$ de lanceurs de javelot parmi les meilleurs du monde. Ces résultats divergent de ceux concernant nos lanceurs de javelot qui 
présentent une valeur moyenne de masse grasse $(8,75 \%)$ inférieure à la valeur moyenne de masse osseuse $(14,73 \%)$.

Nous avons constaté, à travers nos résultats, que les lanceurs de javelot de notre échantillon, par rapport aux autres groupes de lancers, présentaient le pourcentage de masse musculaire le plus élevé et au contraire, présentaient le pourcentage de masse grasse le moins élevé. Cela pourrait s'expliquer par le fait que le lancer de javelot est la seule spécialité de lancers athlétiques qui utilise une course d'élan pour atteindre une vitesse optimale assez élevée, ce qui exige du lanceur de javelot d'avoir peu de masse grasse qui est une charge additionnelle inutile lors du déplacement. 


\section{Conclusion :}

Il se dégage de cette étude plusieurs résultats intéressants :

- Les lanceurs de marteau algériens présentent certaines particularités les caractérisant par rapport aux autres lancers. En effet, ce groupe de lanceurs présente la valeur moyenne de masse corporelle la plus élevée de l'échantillon, avec des valeurs de pourcentage de masse grasse, significativement plus élevés que les autres spécialités de lancer, mais aussi, avec des valeurs de pourcentage de masse musculaire significativement moins élevées que les groupes de lanceurs de disque et de javelot.

- Ces résultats reflétant une certaine surcharge pondérale chez les lanceurs de marteau algériens, il serait intéressant lors de futures études de chercher à déterminer si cet aspect de la morphologie présente un avantage pour la performance dans ce type de lancer, ou au contraire, limiterait la performance et dans ce cas, déterminer si cet excès de poids serait en rapport, par exemple, avec un régime alimentaire inapproprié ou une charge d'entraînement insuffisante.

- Les lanceurs de javelot algériens présentent également certaines particularités par rapport aux autres lancers. Ils présentent les valeurs de masse corporelle et de masse grasse les moins élevées de notre échantillon (spécialement en comparaison avec les lanceurs de marteau), avec le 
pourcentage de masse musculaire le plus élevé (spécialement par rapport au lanceurs de disque et de poids).

- Par rapport à ces particularités, aux caractéristiques techniques du lancer de javelot par rapport aux autres types de lancers (engin le plus léger et existence d'une course d'élan), ajouté aux fait que ce groupe de lanceurs, est le plus jeune de notre échantillon, nous pensons que ce groupe de lanceurs de javelot pourrait représenter un échantillon intéressant pour des études futures. 


\section{Bibliographie :}

(1) BARBE P., RITZ P. (2005). Composition corporelle. Cahier de nutrition et de diététique.40.3.

(2) COH M., MILANOFIC D., EMBERSIC D. (2002). Anthropometric characteristics of elite junior male and female throwers. Collegium antropologicum. Vol 26. pp. 77-83.

(3) COH M., STUHEC S., SUPEJ M. (2008). Comparative biomechanical analysis of the rotational shot put technique. Coll. Antropol. 32. P.249256.

(4) DE GARAY A.L., LEVINE L., CARTER J.E.L. (1974).Genetic and anthropometrical studies of olympic athletes. Academic Prés. New York.

(5) HIRATA K. (1966). Physique and age of Tokyo olympic champions. J. sports med. Phy. Fitness. 6.

(6) KIDD D., WINTER E.M. (1983). Some anthropometric characteristics of the national hammer squad. Br J Sports Med. 17. pp. 152-153.

(7) KRUGER A, DE RIDDER J.H., GROBBELAAR H.W., UNDERHAY C. (2006). A kinanthropometric profile and morphological prediction functions of elite international javelin throwers. In Kinanthropometry IX: Proceedings of the 9th International Conference of the International society for the Advancement of Kinanthropometry. Routledge. New York. p.3848.

(8) MATEIGKA J. (1921). The testing of physical efficiency. American journal of physical anthropology. 4. P. 223-230. 
(9) MAVROEIDI A., STEWARD D. (2003). Prediction of bone, lean and fat tissue mass using dual X-ray absorptiometry as the reference method. In Kinanthropometry VIII, Proceedings of the 8th International Conference of the International society for the Advancement of Kinanthropometry. Edited by Thomas Reilly and Mike Marfell-Jones. Routledge. London. P.26-35.

(10) MORROW J.R., DISCH J.G., WARD J.G., DONOVAN T.J., KATCH F.I. KATCH V.L. WELTMAN A.L. TELLEZ T. (1982). Anthropometric, strength and performance characteristics of American world class throwers. Journal of sports medicine and physical fitness. 1. p. 73-79.

(11) NINDL B.C., FRIEDL K.E., MARCHITELLI L.J., SHIPPEE R.L., THOMAS C.D., PATTON J.F. (1996). Regional fat placement in physically fit males and changes with weight loss. Medicine and science in sports and exercice. 28. P.786-793.

(12) PLATONOV V.N. (1984). L'entraînement sportif, théorie et méthodologie. Editions Revue EPS. Paris.

(13) SCHURCH P. (1984). Perspective et limites du sport de haut niveau vu sous l'angle médical. Revue Macolin. Suisse.

(14) SPENST L.F., MARTIN A.D., DRINKWATER D.T. (1993). Muscle mass of competitive male athletes. Journal of sports sciences. 11. P.3-8.

(15) TANNER J.M. (1964). The physique of olympic athlets. George Allen and Unwin. London.

(16) WILMORE J.H. (1983). Body composition in sport and exercice: directions for future reseach. Medicine ans science in sports ans exercice. 15. P.21-31. 